\title{
Usefulness of Serial Multiorgan Point-of-Care Ultrasound in Acute Heart Failure: Results from a Prospective Observational Cohort
}

\author{
Marta Torres-Arrese 1, Gonzalo García de Casasola-Sánchez ${ }^{1}$, Manuel Méndez-Bailón ${ }^{2}$, Esther Montero-Hernán- \\ dez ${ }^{3}$, Marta Cobo-Marcos ${ }^{4}$, Mercedes Rivas-Lasarte ${ }^{4}$, Luis Caurecel-Díaz ${ }^{5}$, Pablo Rodríguez-Fuertes ${ }^{6}$, Tomas Vi- \\ llén-Villegas ${ }^{7}$ and Yale Tung-Chen ${ }^{3,8, *}$
}

1 Department of Emergency Medicine, Hospital Universitario Fundación de Alcorcón, 28922, Alcorcón, Madrid, Spain; martatorresarrese@gmail.com (MTA); ggcasasolaster@gmail.com (GGC-S)

2 Department of Internal Medicine, Hospital Clínico San Carlos, 28040, Madrid, Spain manuelmenba@hotmail.com (MMB)

3 Department of Internal Medicine, Hospital Universitario Puerta de Hierro, Majadahonda, 28222 Madrid, Spain; esthermhdez@hotmail.com (EMH); yale.tung.chen@gmail.com (YTC)

4 Department of Cardiology, Hospital Universitario Puerta de Hierro, Majadahonda, 28222 Madrid, Spain; martacobomarcos@hotmail.com (MCM); rivaslasarte@gmail.com (MRL)

5 Department of Palliative Medicine, Hospital 12 de Octubre, 28041, Madrid, Spain; luisbalbino.caurcel@salud.madrid.org (LCD)

6 Department of Emergency Medicine, Hospital Universitario La Paz, 28046, Madrid, Spain; pablo.rodriguezfuertes@gmail.com (PRF)

7 Department of Medicine, Universidad Francisco de Vitoria, 28223, Pozuelo de Alarcón, Madrid, Spain; tomasvillen@gmail.com

8 Department of Medicine, Universidad Alfonso X, 28691, Villanueva de la Cañada, Madrid, Spain.

* Correspondence: yale.tung.chen@gmail.com; Tel.: +34-(676)-030-131

\begin{abstract}
Background and Objectives: Acute Heart Failure (AHF) is a common disease and a cause of high morbidity and mortality, constituting a major health problem. The main purpose of this study was to determine the impact of multiorgan ultrasound in identifying the pulmonary hypertension $(\mathrm{PH})$ in patients admitted due to $\mathrm{AHF}$, predict the evolution of the disease during hospitalization and identify areas of improvement in the care of patients with AHF. Materials and Methods: Patients were evaluated with a standard exam of lung ultrasound, echocardiography, inferior vena cava (IVC) and femoral, renal, hepatic, portal venous Doppler flow patterns at admission and on the day of discharge. Results: Thirty patients were enrolled during November 2021. The mean age was seventy-nine years (Standard Deviation - SD 13,4). Seven patients (23.3\%) had a renal function worsening. Regarding ultrasound findings, venous excess ultrasonography score (VExUS) score was calculated at admission and at discharge, surprisingly remaining unchanged or even worsened in most of them (21 patients, $70.0 \%)$. The area under the curve for the Lung Score was $83.9 \%(p=0.008)$, obtaining a cutoff value of 10 that showed a sensitivity of $82.6 \%$ and a specificity of $71.4 \%$ in the identification of intermediate and high $\mathrm{PH}$. It was possible to monitor significant changes between both exams on the lung score $(\mathrm{p}<0.001)$, hepatic vein Doppler $(\mathrm{p}<0.001)$, portal vein Doppler $(\mathrm{p}=$ $0.030)$, intra-renal vein Doppler $(p=0.025)$ and VExUS score $(p=0.023)$, remaining similar the femoral vein Doppler $(p=0.177)$ and IVC $(p=0.132)$. Conclusions: Our study results suggest that performing serial multiorgan Point-of-Care ultrasound can help us to better identify high and intermediate probability of PH patients with AHF. Currently proposed multi-organ, venous Doppler scanning protocols, such as the VExUS score, should be further studied in different populations before expanding its use in AHF patients.
\end{abstract}

Keywords: acute heart failure (AHF); venous congestion; Point-of-Care Ultrasound (POCUS); VExUS (venous excess ultrasonography score) 


\section{Introduction}

Acute heart failure (AHF) is a clinical syndrome whose diagnosis is based on the probability of symptoms and signs coupled with ultrasound and the determination of biochemical markers (1). AHF is very frequently linked to high resource utilization, emergency services visits, hospital admissions, and despite this, it presents a non-negligible complication rate, hovering according to the series of almost $15 \%$ mortality and a $32 \%$ of hospital readmissions (2) . Moreover, patients with AHF with pulmonary hypertension $(\mathrm{PH})$ tend to have a worse prognosis (3). $\mathrm{PH}$ may have important consequences such as liver stasis (4), enteropathy (5), encephalopathy (6) and kidney failure $(7,8)$.

Point-of-Care ultrasound (POCUS), performed at the bedside of the patient, allows to determine the probability of $\mathrm{PH}(9,10)$, based on the combination of different echocardiographic parameters; not easy to obtain in real practice and in exacerbated patients $(11,12)$. Therefore, we are in the need of simpler ultrasound parameters that bring us closer to determine the probability of $\mathrm{PH}$ and its systemic consequences (13). Furthermore, $\mathrm{PH}$ can cause a decrease in NT-proBNP levels, a pillar biochemical marker of AHF, challenging the diagnosis.

Traditionally, we have relied on measuring the inferior vena cava (IVC) as a marker of central venous pressure (CVP). However, CVP does not accurately reflect the patient's venous preload or congestion. In $\mathrm{PH}$, certain valvular heart diseases, advanced chronic obstructive pulmonary disease (COPD) associated with $\mathrm{PH}$ or even in young and athletic patients, it is possible to find a dilated IVC without systemic congestion (14).

Currently, there is an increasing use of ultrasound parameters in the systemic congestion based on the vein Doppler assessment, such as the hepatic and portal veins assessment (15-19), described more than 20 years ago, or more recent one, such as the intrarenal vein Doppler (20). Recently, it has been demonstrated to be useful when integrated into a multiorgan POCUS protocol, such as the venous excess ultrasonography score (VExUS) in cardiac surgical patients $(13,21)$. To our best knowledge, the formulation of such an approach is in need in hospitalized AHF patients.

\section{Materials and Methods}

This was a prospective study performed in an academic hospital, conducted in accordance with the Declaration of Helsinki, and approved by the Research Ethics Committee of our University Hospital. We obtained informed consent from each patient.

\subsection{Patient Selection}

We included patients admitted to an internal medicine ward due to the main diagnosis of AHF. These patients had to exhibit signs attributable to congestion (any of the following: peripheral edema, ascites, jugular engorgement, crackles on pulmonary auscultation, signs of pulmonary congestion on chest X-ray), dyspnea and a NT-proBNP levels $>1000 \mathrm{pg} / \mathrm{ml}$ at admission.

We excluded patients $<18$ years, with hemodynamic instability (vital compromise) or those who declined to participate. A sample of 30 consecutive patients who met these inclusion criteria were enrolled and prospectively studied.

\subsection{Initial patient assessment}

Demographic data (age, sex, weight), medical history (comorbidities, medications), risk factors for AHF (ie, cardiopulmonary diseases), physical exam (weight, negative balance performed), heart rate (HR), sinus rhythm / atrial fibrillation, laboratory tests (creatinine, urea, hemoglobin, white blood cells, platelets, NT-proBNP at admission and before discharge), chest $\mathrm{X}$-ray were registered.

\subsection{Ultrasound data collection}

Patients underwent a multiorgan ultrasound study in the first 24 hours of admission and the same day of discharge. 
We collected the different ultrasound parameters that could be associated to volume overload, the diameters of the IVC, the number of lung B-lines (Lung Score), echocardiographic findings (left ventricular diastolic diameter, left ventricular systolic diameter, interventricular septum and posterior wall thickness in diastole and systole, left ventricular ejection fraction, left and right atrial area, transmitral filling pattern, basal diameter of the right ventricle in apical plane, TAPSE, tricuspid regurgitation velocity, pulmonary artery diameter, right ventricle outflow acceleration time, pulmonary regurgitation velocity , presence of moderate or severe valvular heart disease), intra-renal Doppler ultrasound (polyphasic, biphasic, monophasic), portal vein Doppler ultrasound (pulsatility index $<30 \%, 30-49 \%$, > 50\%), hepatic vein Doppler ultrasound (venous flow pattern type S $>$ D, S $<\mathrm{D}, \mathrm{S}$ wave inversion), femoral vein Doppler ultrasound (pulsatility index $<30 \%, 30-49 \%$, > $50 \%)$.

The exam was performed by an ultrasound fellowship-trained internal medicine physician, who had a long-standing experience in cardiac, vascular and lung US (more than 5 years). A Mindray M7 diagnostic ultrasound system with Phased Array, Curvilinear and linear transducer (Mindray España, Madrid, Spain) and a Kosmos ultrasound handheld device (EchoNous, WA, USA) were used in the study.

The sonographer was blinded to the patient's past medical history, vital signs, symptoms, laboratory measurements, and therapy.

\subsection{Outcome measures and definitions}

Our study aims to determine the impact on accuracy of currently proposed multiorgan, venous Doppler scanning protocols, such as the VExUS score (13), in determining the probability of $\mathrm{PH}$ and the presence of developing renal failure during the hospitalization.

We defined worsening renal failure as a $25 \%$ increase in baseline serum creatine or an increase of $0.3 \mathrm{mg} / \mathrm{dL}$ during hospitalization.

The EVEREST grading score (22), as a clinical course marker of congestion during hospitalization, was calculated for each patient, at admission and at discharge.

Our hypothesis is that multiorgan ultrasound would help us to better identify high and intermediate probability of $\mathrm{PH}$ patients with AHF. Likewise, we intended to make a prediction of the evolution of the disease during hospitalization and identify areas of improvement in the care of patients with AHF.

\subsection{Statistical analysis}

Baseline characteristics are presented as mean and standard deviation (SD) for continuous variables and count and proportions for categorical variables.

Comparisons of means were made using T-Student test and Chi-square test or Fisher's exact two-tailed test was used for categorical variables when it was appropriate. A sample of 30 patients was obtained as previously recommended in reliability studies (23).

We assumed an $\alpha$-value of 0.05 for two-sided hypothesis testing. Analyzes were conducted with the statistical IBM SPSS software v25.0 (SPSS Inc., Chicago, IL, USA).

\section{Results}

During November 2021, a total of 39 patients were screened and fulfilled the inclusion criteria (summarized in figure 1 and table 1), 1 patient declined to participate and 8 were excluded after inclusion: 3 patients died, 1 had a cardiac valve replacement, 1 could not finish the exam, and 3 had other diagnosis than AHF (1 had diffuse lung interstitial disease and 2 had pneumonia, without echocardiographic signs of AHF). 30 patients were included into the final analysis. 


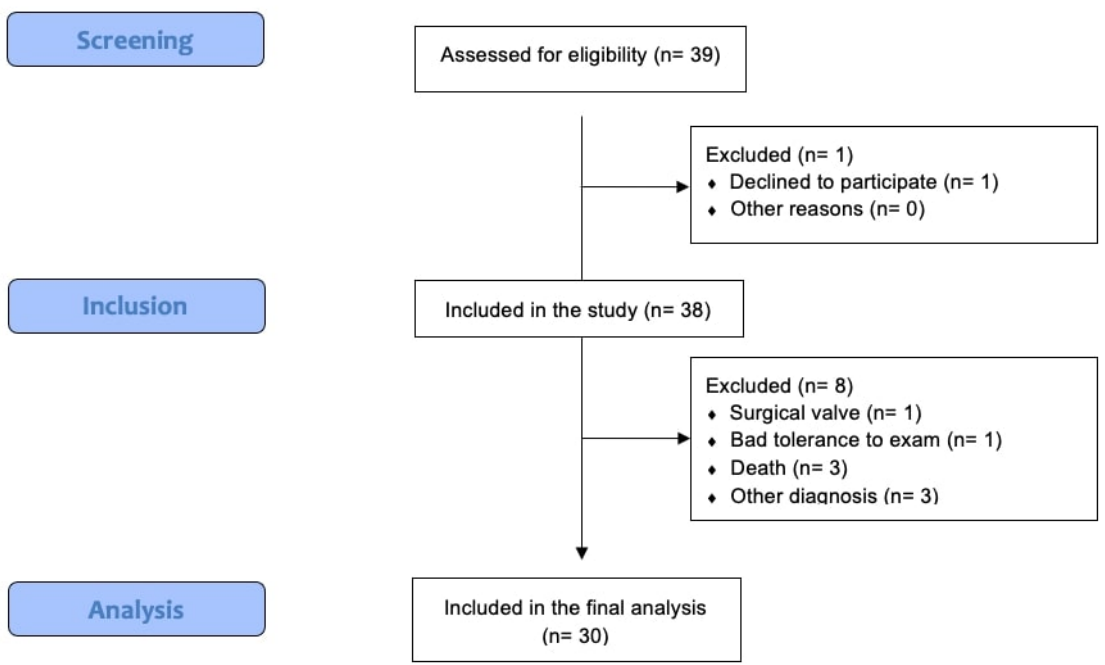

Figure 1. STROBE flow diagram.

The mean age was 79 years (SD 13,4) and 50\% were female. 19 patients $(63.3 \%)$ had an underlying cardiovascular illness. The mean creatinine level was $1.13 \mathrm{mg} / \mathrm{dL}$ (SD 0.5, Normal Value-NV: <0.90) and NT-proBNP was 10846.7 pg / L (SD 11693, NV: <400) at admission. The mean creatinine level was $1.44 \mathrm{mg} / \mathrm{dL}$ (SD 0.8) and NT-proBNP was $6987.3 \mathrm{pg} / \mathrm{L}$ (SD 8999.1) at discharge. 7 patients (23.3\%) had a renal function worsening. Regarding the multiorgan ultrasound exam performed (figure 2), all patients had at least two exams performed, at admission and at discharge (see Table 1). It was possible to monitor significant changes between both exams on the lung score $(p<0.001)$, hepatic vein Doppler profile $(\mathrm{p}<0.001)$, portal vein Doppler $(\mathrm{p}=0.030)$, intra-renal vein Doppler $(\mathrm{p}=$ $0.025)$ and VExUS score $(p=0.023)$, remaining similar to the femoral vein Doppler $(p=$ $0.177)$ and $\operatorname{IVC}(\mathrm{p}=0.132)$.

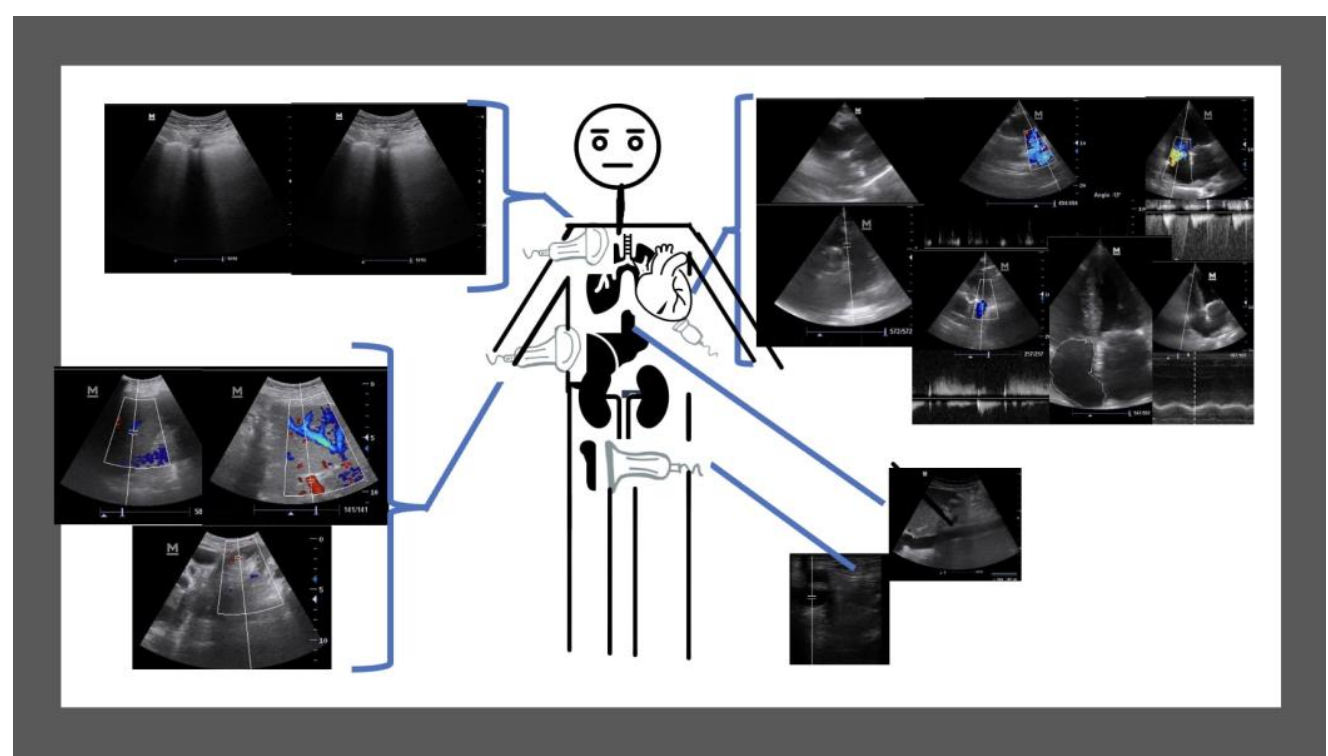

Figure 2. Representation of the ultrasound exam performed in all patients ( $N=30)$.

Table 1. Demographics and clinical characteristics of patients included $(\mathrm{N}=30)$.

\begin{tabular}{lc}
\hline \multicolumn{1}{c}{ Demographics } & N (\%) \\
\hline Gender (female) - N (\%) & $15(50.0)$ \\
Age (years) mean (SD) & $79(13.4)$ \\
\hline
\end{tabular}




\begin{tabular}{|c|c|}
\hline Previolus diseases & $\mathbf{N}(\%)$ \\
\hline Hypertension - N (\%) & $25(83.3)$ \\
\hline Dyslipidemia - N (\%) & $12(40.0)$ \\
\hline Diabetes Mellitus - N (\%) & $8(26.7)$ \\
\hline Chronic Kidney Disease (stage 3 or more) - N (\%) & $8(26.7)$ \\
\hline Previous recent hospitalization - N (\%) & $5(6.7)$ \\
\hline Cardiovascular disease - N (\%) & $19(63.3)$ \\
\hline Atrial Fibrillation - N (\%) & $8(26.7)$ \\
\hline Reduced Ejection Fraction - N (\%) & $3(10.0)$ \\
\hline Pulmonary disease - N (\%) & $9(30.0)$ \\
\hline Phyisical exam & $\mathbf{N}(\%)$ \\
\hline Weight (kg) at admission mean (SD) & $81.1(16.7)$ \\
\hline Weight $(\mathrm{kg})$ at discharge (SD) & $65,6(19.2)$ \\
\hline \multicolumn{2}{|l|}{ Laboratory results - Mean (SD) } \\
\hline NT-proBNP at admission pg/L (SD) & $10846,7(11693,8)$ \\
\hline Urea at admission mg/dL (SD) & $58,3(29,2)$ \\
\hline Sodium at admission mg/dL (SD) & $137,5(6,5)$ \\
\hline Creatinine at admission - mg/dL (SD) & $1,13(0,5)$ \\
\hline Hemoglobin at admission - g/dL (SD) & $12,9(2,4)$ \\
\hline NT-proBNP at discharge pg/L (SD) & $6987,3(8999,1)$ \\
\hline Urea at discharge mg/dL (SD) & $88,7(41,1)$ \\
\hline Sodium at discharge mg/dL (SD) & $141,1(3,9)$ \\
\hline Creatinine at discharge - mg/dL (SD) & $1,44(0,8)$ \\
\hline Hemoglobin at discharge - g/dL (SD) & $12,9(2,2)$ \\
\hline Ultrasound exam - at admission & $\mathbf{N}(\%)$ \\
\hline \multicolumn{2}{|l|}{ Heart rhythm during ultrasound exam } \\
\hline Sinusal rhythm & $10(33.3)$ \\
\hline Atrial fibrillation & $18(60)$ \\
\hline Atrial flutter & $2(6.7)$ \\
\hline Inferior Vena Cava of $>2,1 \mathrm{~cm}$ and $<50 \%$ of collapsability & $12(40,0)$ \\
\hline Inferior Vena Cava of $<2,1 \mathrm{~cm}$ and $>50 \%$ of collapsability & $9(30,0)$ \\
\hline Lung score at admission - mean (SD) & $16,5(9,2)$ \\
\hline \multicolumn{2}{|l|}{ Tricuspid regurgitation } \\
\hline Moderate tricuspid regurgitation - N (\%) & $6(20.0)$ \\
\hline Severe tricuspid regurgitation - N (\%) & $9(30.0)$ \\
\hline Pericardial effusion - N (\%) & $4(13,3)$ \\
\hline Low TAPSE - N (\%) & $11(36,7)$ \\
\hline Mildly reduced Ejection Fraction - N (\%) & $5(16,7)$ \\
\hline
\end{tabular}


Reduced Ejection Fraction - N (\%)

$5(16,7)$

Probability of pulmonary hypertension

Low

Intermediate

$12(40,0)$

High

$11(36,7)$

Hepatic Vein at admission

$\mathrm{S}>\mathrm{D}$

$\mathrm{S}<\mathrm{D}$

$17(56,7)$

S Reversal

$11(36,7)$

Not measurable

Portal Vein at admission

Pulsatility $<30 \%$

Pulsatility $30-50 \%$

$9(40,0)$

Pulsatility $>50 \%$

$6(36,7)$

Intra-Renal Vein at admission

Continuous Monophasic $13(33,3)$

Biphasic flow

$13(43,3)$

Discontinuous Monophasic

$4(13,3)$

Femoral Vein at admission

Pulsatility $<30 \%$

$8(26,7)$

Pulsatility $30-50 \%$

$3(10,0)$

Pulsatility $>50 \%$

$19(63,3)$

\begin{tabular}{lc}
\multicolumn{1}{c}{ Pulsatility $>50 \%$} & $19(63,3)$ \\
\hline Inferior Vena Cava of $>2,1 \mathrm{~cm}$ and $<50 \%$ of collapsability & N (\%) \\
Inferior Vena Cava of $<2,1 \mathrm{~cm}$ and $>50 \%$ of collapsability & $9(30,0)$ \\
Lung score at discharge - mean (SD) & $9,3(8,1)$ \\
Change in Lung score - mean (SD) & $6,7(10.4)$ \\
Inferior Vena Cava of $>2,1 \mathrm{~cm}$ and $<50 \%$ of collapsability & $8(26,7)$ \\
Inferior Vena Cava of $<2,1 \mathrm{~cm}$ and $>50 \%$ of collapsability & $10(33,3)$ \\
Improve in Inferior Vena Cava - N $(\%)$ & $14(46.7)$ \\
Hepatic Vein at discharge & \\
S > D & $9(30,0)$ \\
S $<$ D & $11(36,7)$ \\
S Reversal & $9(30,0)$ \\
Not measurable & $1(3,3)$ \\
Improve in Hepatic Vein profile - N $(\%)$ & $22(73.3)$ \\
Portal Vein at admission & $23(76,3)$ \\
Pulsatility $<30 \%$ & \\
\hline
\end{tabular}




\section{Pulsatility $30-50 \%$}

Pulsatility $>50 \%$

Improve in Portal Vein profile - N (\%)

Worsening in Portal Vein profile - N (\%)

Intra-Renal Vein at admission

Continuous Monophasic

Biphasic flow

Discontinuous Monophasic

Improve in Intra-Renal Vein profile - N (\%)

Worsening in Intra-Renal Vein profile - N (\%)

Femoral Vein at admission

Pulsatility $<30 \%$

Pulsatility $30-50 \%$

Pulsatility $>50 \%$

Improve in VExUS score - N (\%)

Worsening in VExUS score - N (\%)

VExUS score unchanged - $\mathrm{N}(\%)$

Improve in Femoral Vein profile - N (\%)

Worsening in Femoral Vein profile - N (\%)

Follow-up

EVEREST score at admission

EVEREST score at discharge

NYHA at admission

NYHA I

NYHA II

NYHA III

NYHA IV

NYHA at discharge

NYHA I

NYHA II

NYHA III

NYHA IV
$3(10,0)$

$4(13,3)$

$9(30.0)$

$2(6,7)$

$17(56,7)$

$9(30,0)$

$4(13,3)$

$6(20.0)$

$4(13,3)$

$13(43,3)$

$16(53,3)$

$9(30.0)$

14 (46.7)

$9(30.0)$

$3(10,0)$

$10,1(3,1)$

$0,7(0,8)$

$9(30.0)$

18 (60.0)

2 (6.7)

$21(70,0)$

NT-proBNP: NT-proB-type Natriuretic Peptide; NYHA: New York Heart Association; SD: standard deviation; VExUS: venous excess ultrasonography score.

Probability of $\mathrm{PH}$ was calculated $(9,10)$, resulting in a low probability in 7 patients (23.3\%), intermediate in $12(40 \%)$ and high in $11(36.7 \%)$. VExUS score was also calculated at admission and at discharge (table 2), with an improvement in the score in only 9 patients (30\%) and unchanged or worsening in most of them (21 patients, $70.0 \%)$. We calculated the EVEREST score at admission (10.1, SD 3.1) and discharge (0.74, SD 0.8). This score had a low to moderate correlation with VEXUS at admission $(0.532 ; \mathrm{p}=0.004)$, similar to hepatic vein $(0.470 ; \mathrm{p}=0.011)$, portal vein $(0.478 ; \mathrm{p}=0.012)$, intra-renal vein $(0.429$; 
$\mathrm{p}=0.12)$ Doppler at admission. Regarding the EVEREST score at discharge, it was possible to correlate it to the VEXUS score at discharge $(0.461 ; p=0.015)$, Portal vein $(0.675 ; p$ $<0.001)$, intra-renal vein $(0.549 ; \mathrm{p}=0.003)$ and femoral vein Doppler $(0.510 ; \mathrm{p}=0.007)$.

Table 2. Changes between ultrasound parameters at admission and discharge of patients included $(\mathrm{N}=30)$.

\begin{tabular}{|c|c|c|c|}
\hline Ultrasound exam & At admission & At discharge & $p$-Value \\
\hline Inferior Vena Cava of $>2,1 \mathrm{~cm}$ and $<50 \%$ of collapsibility $-\mathrm{N}(\%)$ & $12(40.0)$ & $12(40.0)$ & 0.132 \\
\hline Inferior Vena Cava of $<2,1 \mathrm{~cm}$ and $>50 \%$ of collapsibility $-\mathrm{N}(\%)$ & $9(30.0)$ & $9(30.0)$ & 0.132 \\
\hline Lung score - mean (SD) & $16.5(9.2)$ & $9.3(8.1)$ & $<0.001$ \\
\hline \multicolumn{4}{|l|}{ Hepatic Vein } \\
\hline$S>D-N(\%)$ & $1(3.3)$ & $9(30.0)$ & \multirow{4}{*}{$<0.001$} \\
\hline $\mathrm{S}<\mathrm{D}-\mathrm{N}(\%)$ & $17(56.7)$ & $11(36.7)$ & \\
\hline S Reversal - N (\%) & $11(36.7)$ & $9(30.0)$ & \\
\hline Not measurable $-\mathrm{N}(\%)$ & $1(3.3)$ & $1(3.3)$ & \\
\hline \multicolumn{4}{|l|}{ Portal Vein } \\
\hline Pulsatility $<30 \%-\mathrm{N}(\%)$ & $15(23.3)$ & $23(76.3)$ & \multirow{3}{*}{0.030} \\
\hline Pulsatility $30-50 \%-\mathrm{N}(\%)$ & $9(40.0)$ & $3(10.0)$ & \\
\hline Pulsatility $>50 \%-N(\%)$ & $6(36.7)$ & $4(13.3)$ & \\
\hline \multicolumn{4}{|l|}{ Intra-Renal Vein } \\
\hline Continuous Monophasic - N (\%) & $13(33.3)$ & $17(56.7)$ & \multirow{3}{*}{0.025} \\
\hline Biphasic flow - N (\%) & $13(43.3)$ & $9(30.0)$ & \\
\hline Discontinuous Monophasic - N (\%) & $4(13.3)$ & $4(13.3)$ & \\
\hline \multicolumn{4}{|l|}{ VExUS score } \\
\hline $0-\mathrm{N}(\%)$ & $8(26.7)$ & $10(33.3)$ & \multirow{4}{*}{0.023} \\
\hline $1-\mathrm{N}(\%)$ & $11(36.7)$ & $9(30.0)$ & \\
\hline $2-\mathrm{N}(\%)$ & $6(20.0)$ & $7(23.3)$ & \\
\hline $3-\mathrm{N}(\%)$ & $5(16.7)$ & $4(13.3)$ & \\
\hline \multicolumn{4}{|l|}{ Femoral Vein } \\
\hline Pulsatility <30\% - N (\%) & $8(26.7)$ & $13(43.3)$ & \multirow{3}{*}{0.177} \\
\hline Pulsatility $30-50 \%-\mathrm{N}(\%)$ & $3(10.0)$ & $1(3.3)$ & \\
\hline Pulsatility >50\% - N (\%) & $19(63.3)$ & $16(53.3)$ & \\
\hline
\end{tabular}

NT-proBNP: NT-proB-type Natriuretic Peptide; SD: standard deviation; VExUS: venous excess ultrasonography score.

Regarding NYHA status, most of the patients were in a functional status of NYHA III (18 patients, 60\%) at admission and NYHA I (21 patients, 70\%) at discharge.

Receiver operating characteristic (ROC) curve was calculated for predicting intermediate and high probability of PH according to VExUS score, vein Doppler ultrasound (hepatic, portal, intra-renal and femoral), lung score (figure 3a). The area under the curve (AUC) for the Lung Score was $83.9 \%(p=0.008)$, obtaining a cutoff value of 10 that showed a sensitivity of $82.6 \%$ and a specificity of $71.4 \%$. Followed by VExUS score (AUC $80.1 \%$; $p$ $=0.017$ ) and Hepatic vein Doppler (AUC 79.5\%; $\mathrm{p}=0.020$ ).

The ROC curve was calculated for predicting the probability of renal failure during hospitalization without any significant results (figure $3 \mathrm{~b}$ ). 


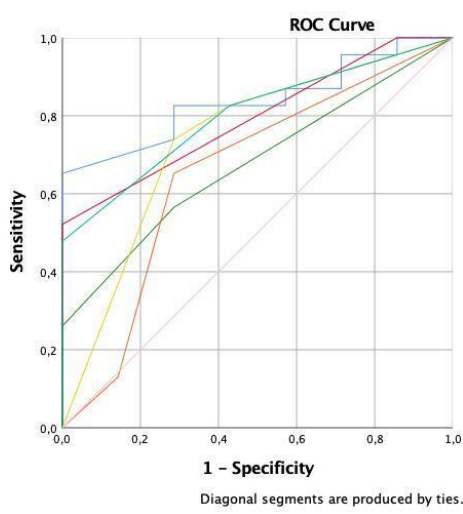

(a)
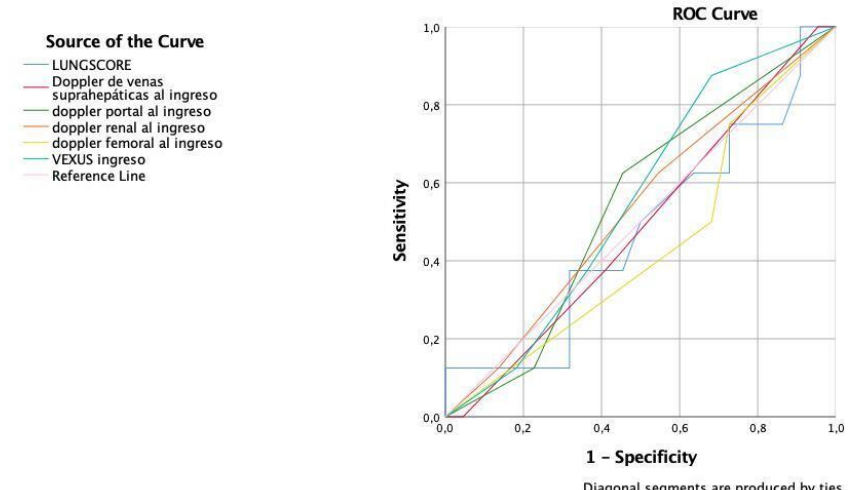

Diagonal segments are produced by ties.

(b)

Figure 3. Receiver operating characteristic (ROC) curve for predicting intermediate and high probability of Pulmonary Hypertension $(\mathrm{PH})$ according to Lung Score (area under the curve - AUC - 83.9\%; $p=0.008$ ), VExUS score (AUC $80.1 \%$; $p$ $=0.017$ ) and Hepatic vein Doppler (AUC 79.5\%; $=0.020) ;(b)$ receiver operating characteristic (ROC) curve for predicting the probability of creatinine worsening during hospitalization, without any significant results (AUC between $47.4 \%$ to $55.4 \%)$.

\section{Discussion}

The importance of venous congestion lies in its correlation with adverse events (10). Traditionally measured with the IVC, its use is endorsed by the European heart failure guidelines (1), however of restricted use due to its numerous limitations (14). Easy to access and reliable diagnostic methods of venous congestion which can accurately guide management in AHF are vital.

Beaubien-Souligny proposes a venous congestion quantification system called the VExUS protocol through hepatic, portal and intra-renal venous Doppler assessment, which seems to be useful in the postoperative cardiac surgery patient to predict renal failure $(13,21)$. However, in acute heart failure, there is an underestimation of creatinine at the beginning of the disease due to hemodilution, so it is not a parameter we must base therapeutic changes (1). As seen in our cohort, there was a worsening in the creatinine levels at discharge, because of hemoconcentration due to the therapy, as well as weight loss and improve in the NYHA status.

In our study, we showed that several ultrasound parameters might be useful for monitoring the intrahospital course of the disease, such as the Lung score, VExUS score and hepatic, portal, intra-renal vein Doppler flow. However, unexpectedly, the dynamics of the VExUS score on admission and discharge remained the same or even disimproved. This raises the question whether the VExUS might be a good parameter, time consuming and cost-effective, to guide the adjustment of the therapy in AHF patients.

In previous studies, left heart failure is more efficiently diagnosed with the Lung score than chest X-ray and NT-proBNP (2); comparable to our findings, Lung score and VExUS score correlated very good with an intermediate and high probability of $\mathrm{PH}$, pointing out that could be a first-line diagnostic tool alternative to advanced echocardiographic studies. Therefore, this group believes, that one of the potential roles of the VExUS protocol is aiding in the diagnosis of PH in AHF, since its diagnosis is complicated and many times we are unable to acquire all the parameters to calculate its probability (3).

Moreover, some exams included in the VExUS protocol, showed in isolation a good correlation with the probability of $\mathrm{PH}$, such as the hepatic vein or in the monitoring of the evolution, such as the portal vein, suggesting being reasonable to adopt different flexible approaches. Additionally, the hepatic veins in patients with rhythm disorders or with intracardiac devices might be difficult to interpret (24). Therefore selecting patients with a portal determination could be sufficient, as supported by other studies (16-19). Regarding, intra-renal Doppler, some observational studies pointed out that severe congestion (monophasic pattern) was associated with an adverse prognosis in $\operatorname{AHF}(25,26)$. However, 
obtaining adequate images can be challenging, especially in the tachypneic patient and has not been studied in patients with structural abnormalities or chronic kidney disease (27).

The femoral vein is an attractive and rapid method for the detection of $\mathrm{PH}$ and right ventricular failure as proposed by Denault (32) through the assessment of the pulsatility, retrograde flow and the absence of respiratory fasicity $(14,32)$; but in our study it seems an insufficient method to be used in isolation. This could be in part owing that our patients could not be positioned in supine ulna (orthopnea), as described in previous studies $(14,32)$. As in our study, we found that the femoral vein could have a similar role as the IVC.

To our best of our knowledge, our study is the first to assess the evolution of the different multi-organ venous Doppler flows in patients with AHF.

We acknowledge some study limitations. First, a small sample of AHF patients, the expert sonographer performed all ultrasound scans on patients consecutively admitted to our department, which limits the generalizability of our results, and ought to be validated in future studies. Second, our study did not analyze the dynamic changes according to the therapy received in different stages, and the patient outcomes, which would have a higher clinical impact. Therefore, for this purpose, we suggest the study can be considered hypothesis generating.

Another limitation is the poor performance of VExUS (and any other ultrasound parameters) in predicting the renal function deterioration, therefore the results from this study provides insights on the need to search other meaningful outcomes, and an opportunity to further investigate the role of ultrasound in this prevalent disease.

\section{Conclusions}

In conclusion, our study suggests that performing serial multiorgan Point-of-Care ultrasound can help us to better identify high and intermediate probability of Pulmonary Hypertension patients with Acute Heart Failure. Currently proposed multi-organ, venous Doppler scanning protocols, such as the VExUS score, should be further studied in different populations before expanding its use.

Author Contributions: Conception and design: YTC, MTA. Analysis and interpretation: YTC. Data collection: MTA. Writing the article: YTC, MTA. Critical revision of the article: YTC, GGdCS, MMB, EM, MCM, MTA, MRL, LCD, PRF, TVV. Final approval of the article: YTC, GGdCS, MMB, EM, MCM, MTA, MRL, LCD, PRF, TVV. Statistical analysis: YTC. Overall responsibility: YTC, MTA.

Funding: This research received no external funding

Institutional Review Board Statement: In this section, please add the Institutional Review Board

Institutional Review Board Statement: The study was conducted according to the guidelines of the Declaration of Helsinki, and approved by the Institutional Review Board of Hospital Universitario Puerta de Hierro (protocol code MIR/HPDH_2021_02 and date of approval July 2021).

Informed Consent Statement: Informed consent was obtained from all subjects involved in the study.

Data Availability Statement: The authors confirm that the data supporting the findings of this study are available from the corresponding author, upon reasonable request.

Conflicts of Interest: The authors declare no conflict of interest.

\section{References}

1. McDonagh TA, Metra M, Adamo M, Gardner RS, Baumbach A, Böhm M, et al. 2021 ESC Guidelines for the diagnosis and treatment of acute and chronic heart failure. Eur Heart J. 2021 Sep 21; 42 (36): 3599-726.

2. Pivetta E, Goffi A, Nazerian P, Castagno D, Tozzetti C, Tizzani P, et al. Lung ultrasound integrated with clinical assessment for the diagnosis of acute decompensated heart failure in the emergency department: a randomized controlled trial. Eur J Heart Fail. 2019 Jun; 21 (6): 754-66.

3. Gorter TM, van Veldhuisen DJ, Bauersachs J, Borlaug BA, Celutkiene J, Coats AJS, et al. Right heart dysfunction and failure in heart failure with preserved ejection fraction: mechanisms and management. Position statement on behalf of the Heart Failure 
Association of the European Society of Cardiology: Right ventricular function in HFpEF. Eur J Heart Fail. 2018 Jan; 20 (1): $16-$ 37.

4. Cubo-Romano P, Torres-Macho J, Soni NJ, Reyes LF, Rodríguez-Almodóvar A, Fernández-Alonso JM, et al. Admission inferior vena cava measurements are associated with mortality after hospitalization for acute decompensated heart failure. J Hosp Med. 2016 Nov; 11 (11): 778-84.

5. Brunkhorst FM. Endotoxins in chronic heart failure. The Lancet. 1999 Aug; 354 (9178): 599.

6. Benkreira A, Beaubien-Souligny W, Mailhot T, Bouabdallaoui N, Robillard P, Desjardins G, et al. Portal Hypertension Is Associated With Congestive Encephalopathy and Delirium After Cardiac Surgery. Can J Cardiol. 2019 Sep; 35 (9): $1134-41$.

7. Spiegel R, Teeter W, Sullivan S, Tupchong K, Mohammed N, Sutherland M, et al. The use of venous Doppler to predict adverse kidney events in a general ICU cohort. Crit Care Lond Engl. 2020 Oct 19; 24 (1): 615.

8. Rosenkranz S, Howard LS, Gomberg-Maitland M, Hoeper MM. Systemic Consequences of Pulmonary Hypertension and RightSided Heart Failure. Circulation. 2020 Feb 25; 141 (8): 678-93.

9. Rivas-Lasarte M, Álvarez-García J, Fernández-Martínez J, Maestro A, López-López L, Solé-González E, et al. Lung ultrasoundguided treatment in ambulatory patients with heart failure: a randomized controlled clinical trial (LUS-HF study). Eur J Heart Fail. 2019 Dec; 21 (12): 1605-13.

10. Prowle JR, Chua H-R, Bagshaw SM, Bellomo R. Clinical review: Volume of fluid resuscitation and the incidence of acute kidney injury - a systematic review. Crit Care. 2012; 16 (4): 230.

11. Pellicori P, Carubelli V, Zhang J, Castiello T, Sherwi N, Clark AL, et al. IVC diameter in patients with chronic heart failure: relationships and prognostic significance. JACC Cardiovasc Imaging. 2013 Jan; 6 (1): 16-28.

12. Kircher BJ, Himelman RB, Schiller NB. Noninvasive estimation of right atrial pressure from the inspiratory collapse of the inferior vena cava. Am J Cardiol. 1990 Aug 15; 66 (4): 493-6.

13. Beaubien-Souligny W, Rola P, Haycock K, Bouchard J, Lamarche Y, Spiegel R, et al. Quantifying systemic congestion with PointOf-Care ultrasound: development of the venous excess ultrasound grading system. Ultrasound J. 2020 Dec; 12 (1): 16.

14. Via G, Tavazzi G, Price S. Ten situations where inferior vena cava ultrasound may fail to accurately predict fluid responsiveness: a physiologically based point of view. Intensive Care Med. 2016 Jul; 42 (7): 1164-7.

15. Abu-Yousef MM. Normal and respiratory variations of the hepatic and portal venous duplex Doppler waveforms with simultaneous electrocardiographic correlation. J Ultrasound Med. 1992 Jun; 11 (6): 263-8.

16. Catalano D, Caruso G, DiFazzio S, Carpinteri G, Scalisi N, Trovato GM. Portal vein pulsatility ratio and heart failure. J Clin ULTRASOUND. 1998; 26 (1): 5.

17. Goncalvesova E, Lesny P, Luknar M, Solik P, Varga I. Changes of portal flow in heart failure patients with liver congestion. Bratisl Lek Listy. 2010; 111 (12): 635-9.

18. Rengo C, Brevetti G, Sorrentino G, D'Amato T, Imparato M, Vitale DF, et al. Portal vein pulsatility ratio provides a measure of right heart function in chronic heart failure. Ultrasound Med Biol. 1998 Mar; 24 (3): 327-32.

19. Hu J-T, Yang S-S, Lai Y-C, Shih C-Y, Chang C-W. Percentage of peak-to-peak pulsatility of portal blood flow can predict rightsided congestive heart failure. World J Gastroenterol. 2003 Aug; 9 (8): 1828-31.

20. de la Espriella-Juan R, Núñez E, Miñana G, Sanchis J, Bayés-Genís A, González J, et al. Intrarenal venous flow in cardiorenal syndrome: a shining light into the darkness. ESC Heart Fail. 2018 Dec; 5 (6): 1173-5.

21. Rola P, Miralles-Aguiar F, Argaiz E, Beaubien-Souligny W, Haycock K, Karimov T, et al. Clinical applications of the venous excess ultrasound (VExUS) score: conceptual review and case series. Ultrasound J. 2021 Dec; 13 (1): 32.

22. Ambrosy AP, Pang PS, Khan S, Konstam MA, Fonarow GC, Traver B, et al. Clinical course and predictive value of congestion during hospitalization in patients admitted for worsening signs and symptoms of heart failure with reduced ejection fraction: findings from the EVEREST trial. Eur Heart J. 2013 Mar 1; 34 (11): 835-43.

23. Koo TK, Li MY. A Guideline of Selecting and Reporting Intraclass Correlation Coefficients for Reliability Research. J Chiropr Med. 2016 Jun; 15 (2): 155-63.

24. 24. Fadel BM, Mohty D, Husain A, Alassas K, Echahidi N, Dahdouh Z, et al. Spectral Doppler of the Hepatic Veins in Rate, Rhythm, and Conduction Disorders. Echocardiography. 2016 Jan; 33 (1): 136-40.

25. Yoshihisa A, Watanabe K, Sato Y, Ishibashi S, Matsuda M, Yamadera Y, et al. Intrarenal Doppler ultrasonography reflects hemodynamics and predicts prognosis in patients with heart failure. Sci Rep. 2020 Dec 17; 10 (1): 22257.

26. Iida N, Seo Y, Sai S, Machino-Ohtsuka T, Yamamoto M, Ishizu T, et al. Clinical Implications of Intrarenal Hemodynamic Evaluation by Doppler Ultrasonography in Heart Failure. JACC Heart Fail. 2016 Aug; 4 (8): 674-82.

27. Bateman GA, Cuganesan R. Renal vein Doppler sonography of obstructive uropathy. AJR Am J Roentgenol. 2002 Apr; 178 (4): 921-5. 